\title{
Endocrine surgery: an update
}

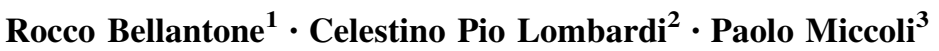

Published online: 16 June 2017

(C) Italian Society of Surgery (SIC) 2017

The endocrine diseases are nowadays a large part of surgical operations worldwide, above all thyroid operations that require not only surgical skill, but also a specific culture to offer the patient the best treatment, tailored surgery, high cure rate with lower morbidities.

In Italy, more than 40,000 thyroidectomies are yearly performed, mostly in about $80 \%$ of centers, performing no more than ten cases per year. In addition, for other gland's pathologies, such as adrenal and parathyroids, the surgical option is spread in many centers.

The editors in chief have so asked us to provide a special monothematic issue, asking remarkable Italian and foreign colleagues to make the up-to-date about particular aspects of this discipline.

Therefore, it is really a great pleasure for all of us to have put together many of experienced colleagues, involved and dedicated to various fields of the endocrine surgical super specialty, to cooperate to this monothematic issues of Updates in Surgery.

Some of the aspects we routinely encounter in our practice, such as indications for surgery, which surgical operation, which approach either open or laparoscopic or robotic, are thoroughly investigated in this issue.

Celestino Pio Lombardi

celestinopio.lombardi@unicatt.it

1 Department of Endocrine and Metabolic Surgery, Catholic University, Policlinico Gemelli, Rome, Italy

2 Department of Endocrine Surgery, Catholic University, Policlinico Gemelli, Rome, Italy

3 Department of Surgical Pathology, University of Pisa, Pisa, Italy
In addition, risk factors, tricks to prevent lesions, or morbidities following are extensively discussed.

We also decided to include some more specific aspects, such as surgical implications of pregnancy in thyroid disease, the role of genetics in hereditary tumors, the extension of the radical surgery in thyroid cancers, and different laparoscopic approaches to the adrenal gland.

Finally, we would express many thanks to our colleagues for the effort to join us in this work and we hope that all together may have contributed to clarify some new aspects and pushed our younger surgeons to study and practice at higher level all the fields of endocrine surgery.

\section{Compliance with ethical standards}

Conflict of interest The authors declare that they have no competing interests.

Research involving human participants and/or animals The present manuscript is compliant with ethical standard. This study does not invole any kind of animal related contacts or experiments by any of the listed authors.

Informed consent All the patients included gave their informed consent. 